\title{
Solution of Some Second Order Ordinary Differential Equations Using a Derived Algorithm
}

\author{
R. B. Ogunrinde, J. O. Olubunmi \\ Department of Mathematical Sciences, Ekiti State University, Ado-Ekiti, Nigeria \\ Email: rowbose@yahoo.com, oluwasanmiolubunmi9@gmail.com
}

How to cite this paper: Ogunrinde, R.B. and Olubunmi, J.O. (2019) Solution of Some Second Order Ordinary Differential Equations Using a Derived Algorithm. Applied Mathematics, 10, 312-325. https://doi.org/10.4236/am.2019.105022

Received: July 6, 2018

Accepted: May 18, 2019

Published: May 21, 2019

Copyright $\odot 2019$ by author(s) and Scientific Research Publishing Inc. This work is licensed under the Creative Commons Attribution International License (CC BY 4.0).

http://creativecommons.org/licenses/by/4.0/

\section{cc) (7) Open Access}

\begin{abstract}
We emphasized explicitly on the derivation and implementation of a new numerical algorithm scheme which gave stable results that show the applicability of the method. In this paper, we aimed to solve some second order initial value problems of ordinary differential equations and compare the results with the theoretical solution. Using this method to solve some initial value problems of second order ordinary differential equations, we discovered that the results compared favorably with the theoretical solution which led to the conclusion that the new numerical algorithm scheme derived in the research is approximately correct and can be prescribed for any related ordinary differential equations.
\end{abstract}

\section{Keywords}

Numerical Scheme, Ordinary Differential Equation, Scheme Development

\section{Introduction}

Numerical methods are methods that are constructed through a given interval. The methods start with an initial point and then take a short step forward in time to find the next solution point. The process then continues with subsequent steps to map out the solution. There are two main numerical methods of solving initial value problems of ordinary differential equations. They are single step methods, also known as one step method and multistep methods. The single-step methods are the method that uses information about the solution at one point $X_{n}$, to advance it to the next point $x_{n+1}$. The single step methods have certain advantages which include, being self-starting and having the flexibility to change step size from one step to the next. 
Various numerical methods have been developed for the solution of some initial value problems of ordinary differential equations. Some of the numerical analysts who have worked extensively on the development on numerical methods are: [1] [2] [3] [4] [5]. Development of a scheme for solving some initial value problem of ordinary differential equations with a particular basis function was carried out by [1] which was improved upon by [2] for solving related problems. [4] and [5] worked extensively in other to improve upon schemes developed by [1] and [2] and better methods were produced. The efficiency of all these contributed efforts in numerical analysis had been measured and tested for their stability, accuracy, convergence and consistency properties [6] [7] [8]. The accuracy properties of different methods are usually compared by considering the order of convergence as well as the truncation error coefficients of the various methods. Research has shown that for a method to be suitable for solving any sets of initial value problems (ivps) in ordinary differential equations (ODEs), it must have all the mentioned characteristics.

Recently [9] developed a scheme in which standard finite difference schemes were developed. Similarly, [4] also worked on some approximation techniques which were used to derive qualitatively stable non-standard finite difference schemes.

In this paper, a new one-step numerical method is developed with the above mentioned characteristics in mind to solve some initial value problems of ordinary differential equations which were based on the local representation of the theoretical solution to initial value problem of the form $y^{\prime}=f(x, y) ; y(a)=\eta$. In the interval $\left(x_{n}, x_{n+1}\right)$ by interpolating function $F(x)=a_{0}+a_{1} x+a_{2} x^{2}+a_{3} x^{3}+b x R_{e}\left(e^{k x+\mu}\right), a_{0}, a_{1}, a_{2}, a_{3}$ and $b$ are real undetermined coefficients and $k, \mu$ are complex parameters. But in this paper, we shall be using the same assumptions but different interpolating functions such as:

$F(x)=a_{0}+a_{1} x+a_{2} x^{2}+a_{3} x^{3}+b x R_{e}\left(e^{k x+\mu}\right)$, where $a_{0}, a_{1}, a_{2}, a_{3}$ and $b$ are real undetermined coefficients and $k, \mu$ are complex parameters.

\section{Methodogy}

Considering an interpolating function:

$$
f(x)=a_{0}+a_{1} x+a_{2} x^{2}+a_{3} x^{3}+b x R_{e}\left(e^{k x+\mu}\right)
$$

where $a_{0}, a_{1}, a_{2}, a_{3}$ and $b$ are real undetermined coefficients and $k, \mu$ are complex parameters.

Since $k$ and $\mu$ are complex parameters, then we have:

$$
k=k_{1}+i k_{2}
$$

Also, $\mu=i \theta$, where $i^{2}=-1$, therefore putting this together with (2) in (1), we have the Interpolating function to be:

$$
f(x)=a_{0}+a_{1} x+a_{2} x^{2}+a_{3} x^{3}+b x e^{k_{1}} x \cos \left(k_{2} x+\sigma\right)
$$

Let us define $R(x)$ and $\theta(x)$ as follows: 


$$
\left.\begin{array}{l}
R(x)=x e^{k_{1} x} \\
\theta(x)=k_{2} x+\sigma
\end{array}\right\}
$$

Putting (4) in (3), we have:

$$
f(x)=a_{0}+a_{1} x+a_{2} x^{2}+a_{3} x^{3}+b R(x) \cos \theta(x)
$$

By assumption, $y_{n}$ is a numerical estimate to the theoretical solution $y\left(x_{n}\right)$ and also $f_{n}=f\left(x_{n}, y_{n}\right)$. Let our mesh points (self length) be define as follows:

$$
x_{n}=a+n h ; n=0,1,2, \cdots, a=0, x_{n}=n h . \quad x_{n+1}=(n+1) h
$$

Imposing the following constraints on the interpolating function (5), we have:

1) The interpolating function must coincide with the theoretical solution at $x=x_{n}$ and $x=x_{n+1}$. This required that:

$$
f\left(x_{n+1}\right)=a_{0}+a_{1} x_{n+1}+a_{2} x_{n+1}^{2}+a_{3} x_{n+1}^{3}+b R\left(x_{n+1}\right) \cos \theta\left(x_{n}\right) .
$$

That is, $f\left(x_{n}\right)=y\left(x_{n}\right)$ and

$$
f\left(x_{n+1}\right)=a_{0}+a_{1} x_{n+1}+a_{2} x_{n+1}^{2}+a_{3} x_{n+1}^{3}+b R\left(x_{n+1}\right) \cos \theta\left(x_{n+1}\right)
$$

It implies that $f\left(x_{n+1}\right)=y\left(x_{n+1}\right)$.

2) The first, second, third and fourth derivatives with respect to $x$ of the interpolating function respectively coincide with the differential equation as well as its first, second, third and fourth derivatives with respect to $x$ at $X_{n}$, i.e.

$$
\left.\begin{array}{l}
F^{1}\left(x_{n}\right)=f_{n} \\
F^{2}\left(x_{n}\right)=f_{n}^{1} \\
F^{3}\left(x_{n}\right)=f_{n}^{2} \\
F^{4}\left(x_{n}\right)=f_{n}^{3}
\end{array}\right\}
$$

From Equation (9) implies:

$$
f^{\prime}\left(x_{n}\right)=f_{n}+2 a_{2} x_{n}+3 a_{3} x_{n}^{2}+\left[\cos \theta\left(x_{n}\right) \frac{\mathrm{d}}{\mathrm{d} x}\left(b R\left(x_{n}\right)\right)+b R\left(x_{n}\right) \frac{\mathrm{d}}{\mathrm{d} x}(\cos \theta(x))\right](
$$

where

$$
\begin{aligned}
\frac{\mathrm{d}}{\mathrm{d} x}\left(b R\left(x_{n}\right)\right) & =\frac{\mathrm{d}}{\mathrm{d} x}\left(b x e^{k_{1} x}\right)=e^{k_{1} x} \cdot b+b x \cdot k_{1} e^{k_{1} x}=b e^{k_{1} x}+b k_{1} x e^{k_{1} x} \\
& =b e^{k_{1} x}+b k_{1} R\left(x_{n}\right) \\
\frac{\mathrm{d}}{\mathrm{d} x} & \cos \theta\left(x_{n}\right) \frac{\mathrm{d}}{\mathrm{d} x}\left[\cos \left(k_{2} x+\sigma\right)\right]=-k_{2} \sin \theta\left(x_{n}\right)
\end{aligned}
$$

Putting (11) \& (12) in (10) we have:

$$
\begin{aligned}
f^{\prime}\left(x_{n}\right)= & f_{n}=a_{1}+2 a_{2} x_{n}+3 a_{3} x_{n}^{2}\left[\cos \theta\left(x_{n}\right)\left\{b e^{k_{1} x}+b k_{1} R\left(x_{n}\right)\right\}\right. \\
& \left.+b R\left(x_{n}\right)\left(-k_{2} \sin \theta\left(x_{n}\right)\right)\right] \\
= & a_{1}+2 a_{2} x_{n}+3 a_{3} x_{n}^{2}+\left[b e^{k_{1} x} \cos \theta\left(x_{n}\right)+b k_{1} R\left(x_{n}\right) \cos \theta\left(x_{n}\right)\right. \\
& \left.-b R\left(x_{n}\right)\left(-k_{2} \sin \theta\left(x_{n}\right)\right)\right] \\
f_{n}= & a_{1}+2 a_{2} x_{n}+3 a_{3} x_{n}^{2}+b\left[e^{k_{1} x_{n}} \cos \theta\left(x_{n}\right)+k_{1} R\left(x_{n}\right) \cos \theta\left(x_{n}\right)\right. \\
- & \left.k_{2} R\left(x_{n}\right) \sin \theta\left(x_{n}\right)\right]
\end{aligned}
$$

That is, $F^{\prime}\left(x_{n}\right)=f_{n}$ 


$$
\begin{aligned}
F^{\prime \prime}\left(x_{n}\right)= & f_{n}^{\prime}=2 a_{2} x_{n}+6 a_{3} x_{n}+b\left[\left(e^{k_{1} x_{n}} \frac{\mathrm{d}}{\mathrm{d} x} \cos \theta\left(x_{n}\right)+\cos \theta\left(x_{n}\right) \frac{\mathrm{d}}{\mathrm{d} x} e^{k_{1} x_{n}}\right)\right. \\
& +\left(k_{1} R\left(x_{n}\right) \frac{\mathrm{d}}{\mathrm{d} x} \cos \theta\left(x_{n}\right)+\cos \theta\left(x_{n}\right) \frac{\mathrm{d}}{\mathrm{d} x} k_{1} R\left(x_{n}\right)\right. \\
& \left.\left.-\left(R\left(x_{n}\right) \frac{\mathrm{d}}{\mathrm{d} x} \sin \theta\left(x_{n}\right)+\sin \theta\left(x_{n}\right) \frac{\mathrm{d}}{\mathrm{d} x} R\left(x_{n}\right)\right)\right)\right]
\end{aligned}
$$

where

$$
\begin{aligned}
k_{1} R\left(x_{n}\right) & =k_{1}\left[x e^{k_{1} x_{n}}\right]=k_{1}\left(x \frac{\mathrm{d}}{\mathrm{d} x} e^{k_{1} x_{n}}+e^{k_{1} x_{n}} \frac{\mathrm{d}}{\mathrm{d} x} x\right) \\
& =k_{1}^{2} x e^{k_{1} x_{n}}+k_{1} e^{k_{1} x_{n}}=k_{1}^{2} R\left(x_{n}\right)+k_{1} e^{k_{1} x_{n}}
\end{aligned}
$$

Since $R\left(x_{n}\right)=x_{n} e^{k_{1}} x_{n}=e^{k_{1} x_{n}}+k_{1} R\left(x_{n}\right)$.

Putting (15) in (14)

$$
\begin{aligned}
& F^{\prime \prime}\left(x_{n}\right)=f_{n}^{\prime}=2 a_{2} x_{n}+6 a_{3} x_{n}+b\left[\left\{e^{k_{1} x_{n}}\left(-\sin \theta\left(x_{n}\right)\right)+\cos \theta\left(x_{n}\right)\left(k_{1} e^{k_{1} x_{n}}\right)\right\}\right. \\
& +k_{1} R\left(x_{n}\right)\left(-\sin \theta\left(x_{n}\right)\right)+\cos \theta\left(x_{n}\right)\left(k_{1}^{2} R\left(x_{n}\right)+k_{1} e^{k_{1} x_{n}}\right) \\
& \left.-\left\{R\left(x_{n}\right) \cos \theta\left(x_{n}\right)+\sin \theta\left(x_{n}\right)\left(e^{k_{1} x_{n}}+k_{1} R\left(x_{n}\right)\right)\right\}\right] \\
& F^{\prime \prime}\left(x_{n}\right)=2 a_{2} x_{n}+6 a_{3} x_{n}+b\left[\left\{-e^{k_{1} x_{n}} \sin \theta\left(x_{n}\right)+k_{1} e^{k_{1} x_{n}} \cos \theta\left(x_{n}\right)\right\}\right. \\
& -k_{1} R\left(x_{n}\right) \sin \theta\left(x_{n}\right)+k_{1}^{2} R\left(x_{n}\right) \cos \theta\left(x_{n}\right)+k_{1} e^{k_{1} x_{n}} \cos \theta\left(x_{n}\right) \\
& \left.-R\left(x_{n}\right) \cos \theta\left(x_{n}\right)-e^{k_{1} x_{n}} \sin \theta\left(x_{n}\right)-k_{1} R\left(x_{n}\right) \sin \theta\left(x_{n}\right)\right] \\
& f_{n}^{\prime}=2 a_{2} x_{n}+6 a_{3} x_{n}+b\left\{-2 e^{k_{1} x_{n}} \sin \theta\left(x_{n}\right)+2 k_{1} e^{k_{1} x_{n}} \cos \theta\left(x_{n}\right)\right. \\
& \left.-2 k_{1} R\left(x_{n}\right) \sin \theta\left(x_{n}\right)+k_{1}^{2} R\left(x_{n}\right) \cos \theta\left(x_{n}\right)-R\left(x_{n}\right) \cos \theta\left(x_{n}\right)\right\} \\
& F^{\prime \prime \prime}\left(x_{n}\right)=f_{n}^{\prime \prime}=6 a_{3}+b\left[-\left\{2 e^{k_{1} x_{n}} \frac{\mathrm{d}}{\mathrm{d} x} \sin \theta\left(x_{n}\right)+\sin \theta\left(x_{n}\right) \frac{\mathrm{d}}{\mathrm{d} x} 2 e^{k_{1} x_{n}}\right\}\right. \\
& +\left\{2 k_{1} e^{k_{1} x_{n}} \frac{\mathrm{d}}{\mathrm{d} x} \cos \theta\left(x_{n}\right)+\cos \theta\left(x_{n}\right) \frac{\mathrm{d}}{\mathrm{d} x} 2 k_{1} e^{k_{1} x_{n}}\right\} \\
& -\left\{2 k_{1} R\left(x_{n}\right) \frac{\mathrm{d}}{\mathrm{d} x} \sin \theta\left(x_{n}\right)+\sin \theta\left(x_{n}\right) \frac{\mathrm{d}}{\mathrm{d} x} 2 k_{1} R\left(x_{n}\right)\right\} \\
& +\left\{k_{1}^{2} R\left(x_{n}\right) \frac{\mathrm{d}}{\mathrm{d} x} \cos \theta\left(x_{n}\right)+\cos \theta\left(x_{n}\right) \frac{\mathrm{d}}{\mathrm{d} x} k_{1}^{2} R\left(x_{n}\right)\right\} \\
& \left.-\left\{R\left(x_{n}\right) \frac{\mathrm{d}}{\mathrm{d} x} \cos \theta\left(x_{n}\right)+\cos \theta\left(x_{n}\right) \frac{\mathrm{d}}{\mathrm{d} x} R\left(x_{n}\right)\right\}\right] \\
& F^{\prime \prime \prime}\left(x_{n}\right)=6 a_{3}+b\left[-\left(2 e^{k_{1} x_{n}} \cos \theta\left(x_{n}\right)+2 k_{1} e^{k_{1} x_{n}} \sin \theta\left(x_{n}\right)\right)\right. \\
& +2 k_{1} e^{k_{1} x_{n}}\left(-\sin \theta\left(x_{n}\right)\right)+2 k_{1}^{2} e^{k_{1} x_{n}} \cos \theta\left(x_{n}\right) \\
& -2 k_{1} R\left(x_{n}\right) \cos \theta\left(x_{n}\right)+\left[2 k_{1} e^{k_{1} x_{n}}+2 k_{1}^{2} R\left(x_{n}\right)\right] \sin \theta\left(x_{n}\right) \\
& +k_{1}^{2} R\left(x_{n}\right)\left(-\sin \theta\left(x_{n}\right)\right)+k_{1}^{2} e^{k_{1} x_{n}}+k_{1}^{3} R\left(x_{n}\right) \cos \theta\left(x_{n}\right) \\
& \left.-R\left(x_{n}\right)\left(-\sin \theta\left(x_{n}\right)\right)+\left(e^{k_{1} x_{n}}+k_{1} R\left(x_{n}\right)\right) \cos \theta\left(x_{n}\right)\right]
\end{aligned}
$$




$$
\begin{aligned}
& F^{\prime \prime \prime}\left(x_{n}\right)=f_{n}^{\prime \prime}=6 a_{3}+b\left[-3 e^{k_{1} x_{n}} \cos \theta\left(x_{n}\right)-6 k_{1} e^{k_{1} x_{n}} \sin \theta\left(x_{n}\right)\right. \\
& +3 k_{1}^{2} R\left(x_{n}\right) \sin \theta\left(x_{n}\right)-2 k_{1} R\left(x_{n}\right) \cos \theta\left(x_{n}\right) \\
& \left.+k_{1}^{3} R\left(x_{n}\right) \cos \theta\left(x_{n}\right)+R\left(x_{n}\right) \sin \theta\left(x_{n}\right)-k_{1} e^{k_{1} x_{n}} \cos \theta\left(x_{n}\right)\right] \\
& F^{\prime v}\left(x_{n}\right)=f^{\prime \prime \prime}\left(x_{n}\right) \\
& =b\left[-3\left\{e^{k_{1} x_{n}} \frac{\mathrm{d}}{\mathrm{d} x} \cos \theta\left(x_{n}\right)+\cos \theta\left(x_{n}\right) \frac{\mathrm{d}}{\mathrm{d} x} e^{k_{1} x_{n}}\right\}\right. \\
& -6\left\{e^{k_{1} x_{n}} \frac{\mathrm{d}}{\mathrm{d} x} \sin \theta\left(x_{n}\right)+\sin \theta\left(x_{n}\right) \frac{\mathrm{d}}{\mathrm{d} x} e^{k_{1} x_{n}}\right\} \\
& +3 k_{1}^{2}\left\{e^{k_{1} x_{n}} \frac{\mathrm{d}}{\mathrm{d} x} \cos \theta\left(x_{n}\right)+\cos \theta\left(x_{n}\right) \frac{\mathrm{d}}{\mathrm{d} x} e^{k_{1} x_{n}}\right\} \\
& -3 k_{1}^{2}\left\{R\left(x_{n}\right) \frac{\mathrm{d}}{\mathrm{d} x} \sin \theta\left(x_{n}\right)+\sin \theta\left(x_{n}\right) \frac{\mathrm{d}}{\mathrm{d} x} R\left(x_{n}\right)\right\} \\
& -2 k_{1}\left\{R\left(x_{n}\right) \frac{\mathrm{d}}{\mathrm{d} x} \cos \theta\left(x_{n}\right)+\cos \theta\left(x_{n}\right) \frac{\mathrm{d}}{\mathrm{d} x} R\left(x_{n}\right)\right\} \\
& +k_{1}^{3}\left\{R\left(x_{n}\right) \frac{\mathrm{d}}{\mathrm{d} x} \cos \theta\left(x_{n}\right)+\cos \theta \frac{\mathrm{d}}{\mathrm{d} x} R\left(x_{n}\right)\right\} \\
& +\left\{R\left(x_{n}\right) \frac{\mathrm{d}}{\mathrm{d} x} \sin \theta\left(x_{n}\right)+\sin \theta\left(x_{n}\right) \frac{\mathrm{d}}{\mathrm{d} x} R\left(x_{n}\right)\right\} \\
& -k_{1}\left\{e^{k_{1} x_{n}} \frac{\mathrm{d}}{\mathrm{d} x} \cos \theta\left(x_{n}\right)+\cos \theta\left(x_{n}\right) \frac{\mathrm{d}}{\mathrm{d} x} e^{k_{1} x_{n}}\right\} \\
& F^{I V}\left(x_{n}\right)=b\left[4 e^{k_{1} x_{n}} \sin \theta\left(x_{n}\right)-11 k_{1} e^{k_{1} x_{n}} \cos \theta\left(x_{n}\right)-12 k_{1}^{2} e^{k_{1} x_{n}} \sin \theta\left(x_{n}\right)\right. \\
& +4 k_{1}^{3} e^{k_{1} x_{n}} \cos \theta\left(x_{n}\right)-5 k_{1}^{2} R\left(x_{n}\right) \cos \theta\left(x_{n}\right)-4 k_{1}^{3} R\left(x_{n}\right) \sin \theta\left(x_{n}\right) \\
& +3 k_{1} R\left(x_{n}\right) \sin \theta\left(x_{n}\right)+R\left(x_{n}\right) \cos \theta\left(x_{n}\right)+k_{1} e^{k_{1} x_{n}} \sin \theta\left(x_{n}\right) \\
& \left.-k_{1}^{2} e^{k_{1} x_{n}} \cos \theta\left(x_{n}\right)+k_{1}^{4} R\left(x_{n}\right) \cos \theta\left(x_{n}\right)\right] \\
& b=\frac{f_{n}^{\prime \prime \prime}}{e^{k_{1} x_{n}}\left\{4 \sin \theta\left(x_{n}\right)-11 k_{1} \cos \theta\left(x_{n}\right)-12 k_{1}^{2} \sin \theta\left(x_{n}\right)+4 k_{1}^{3} \cos \theta\left(x_{n}\right)\right.} \\
& \left.+k_{1} \sin \theta\left(x_{n}\right)-k_{1}^{2} \cos \theta\left(x_{n}\right)\right\}+R\left(x_{n}\right)\left\{-5 k_{1}^{2} \cos \theta\left(x_{n}\right)\right. \\
& \left.-4 k_{1}^{3} \sin \theta\left(x_{n}\right)+3 k_{1} \sin \theta\left(x_{n}\right)+\cos \theta\left(x_{n}\right)+k_{1}^{4} \cos \theta\left(x_{n}\right)\right\}
\end{aligned}
$$

From (17), we have:

$$
\begin{aligned}
a_{3}= & \frac{1}{6}\left[f_{n}^{2}-b\left\{-3 e^{k_{1} x_{n}} \cos \theta\left(x_{n}\right)-6 k_{1} e^{k_{1} x_{n}} \sin \theta\left(x_{n}\right)+3 k_{1}^{2} e^{k_{1} x_{n}} \cos \theta\left(x_{n}\right)\right.\right. \\
& -3 k_{1}^{2} R\left(x_{n}\right) \sin \theta\left(x_{n}\right)-2 k_{1} R\left(x_{n}\right) \cos \theta\left(x_{n}\right)+k_{1}^{3} R\left(x_{n}\right) \cos \theta\left(x_{n}\right) \\
& \left.\left.+R\left(x_{n}\right) \sin \theta\left(x_{n}\right)-k_{1} e^{k_{1} x_{n}} \cos \theta\left(x_{n}\right)\right\}\right]
\end{aligned}
$$

Putting (19) in (20), we have: 


$$
\left.\begin{array}{rl}
a_{3} & =\frac{1}{6}\left[f_{n}^{2}-\left(\begin{array}{l}
\frac{f_{n}^{3}}{e^{k_{1} x_{n}}\left\{\sin \theta\left(x_{n}\right)-11 k_{1} \cos \theta\left(x_{n}\right)-12 k_{1}^{2} \sin \theta\left(x_{n}\right)+4 k_{1}^{3} \cos \theta\left(x_{n}\right)\right.} \\
\left.+k_{1} \sin \theta\left(x_{n}\right)-k_{1}^{2} \cos \theta\left(x_{n}\right)\right\}+R\left(x_{n}\right)\left\{-5 k_{1}^{2} \cos \theta\left(x_{n}\right)\right. \\
\left.-4 k_{1}^{3} \sin \theta\left(x_{n}\right)+3 k_{1} \sin \theta\left(x_{n}\right)+\cos \theta\left(x_{n}\right)+k_{1}^{4} \cos \theta\left(x_{n}\right)\right\}
\end{array}\right)\right. \\
& \times\left\{\begin{array}{l}
-3 e^{k_{1} x_{n}} \cos \theta\left(x_{n}\right)-6 k_{1} e^{k_{1} x_{n}} \sin \theta\left(x_{n}\right)+3 k_{1}^{2} e^{k_{1} x_{n}} \cos \theta\left(x_{n}\right)-3 k_{1}^{2} R\left(x_{n}\right) \sin \theta\left(x_{n}\right) \\
-
\end{array}\right] \\
& \left.2 k_{1} R\left(x_{n}\right) \cos \theta\left(x_{n}\right)+k_{1}^{3} R\left(x_{n}\right) \cos \theta\left(x_{n}\right)+R\left(x_{n}\right) \sin \theta\left(x_{n}\right)-k_{1} e^{k_{1} x_{n}} \cos \theta\left(x_{n}\right)\right\}
\end{array}\right]
$$

Let:

$$
\begin{aligned}
v= & -3 e^{k_{1} x_{n}} \cos \theta\left(x_{n}\right)-6 k_{1} e^{k_{1} x_{n}} \sin \theta\left(x_{n}\right)+3 k_{1}^{2} e^{k_{1} x_{n}} \cos \theta\left(x_{n}\right) \\
& -3 k_{1}^{2} R\left(x_{n}\right) \sin \theta\left(x_{n}\right)-2 k_{1} R\left(x_{n}\right) \cos \theta\left(x_{n}\right)+k_{1}^{3} R\left(x_{n}\right) \cos \theta\left(x_{n}\right) \\
& +R\left(x_{n}\right) \sin \theta\left(x_{n}\right)-k_{1} e^{k_{1} x_{n}} \cos \theta\left(x_{n}\right)
\end{aligned}
$$

Then, (21) becomes:

$$
a_{3}=\frac{1}{6}\left[f_{n}^{2}-\left(\begin{array}{l}
\frac{f_{n}^{3}}{e^{k_{1} x_{n}}\left\{\sin \theta\left(x_{n}\right)-11 k_{1} \cos \theta\left(x_{n}\right)-12 k_{1}^{2} \sin \theta\left(x_{n}\right)+4 k_{1}^{3} \cos \theta\left(x_{n}\right)\right.} \\
\left.+k_{1} \sin \theta\left(x_{n}\right)-k_{1}^{2} \cos \theta\left(x_{n}\right)\right\}+R\left(x_{n}\right)\left\{-5 k_{1}^{2} \cos \theta\left(x_{n}\right)\right. \\
\left.-4 k_{1}^{3} \sin \theta\left(x_{n}\right)+3 k_{1} \sin \theta\left(x_{n}\right)+\cos \theta\left(x_{n}\right)+k_{1}^{4} \cos \theta\left(x_{n}\right)\right\}
\end{array}\right) v\right]
$$

From (16), we have:

$$
\left.\begin{array}{l}
a_{2}=\frac{1}{2}\left[f_{n}^{1}-\left(f_{n}^{2}-\left(\begin{array}{l}
f_{n}^{3} \\
e^{k_{1} x_{n}}\left\{\sin \theta\left(x_{n}\right)-11 k_{1} \cos \theta\left(x_{n}\right)-12 k_{1}^{2} \sin \theta\left(x_{n}\right)+4 k_{1}^{3} \cos \theta\left(x_{n}\right)\right. \\
\left.+k_{1} \sin \theta\left(x_{n}\right)-k_{1}^{2} \cos \theta\left(x_{n}\right)\right\}+R\left(x_{n}\right)\left\{-5 k_{1}^{2} \cos \theta\left(x_{n}\right)\right. \\
\left.-4 k_{1}^{3} \sin \theta\left(x_{n}\right)+3 k_{1} \sin \theta\left(x_{n}\right)+\cos \theta\left(x_{n}\right)+k_{1}^{4} \cos \theta\left(x_{n}\right)\right\}
\end{array}\right) v x_{n}\right.\right. \\
-b\left\{-2 e^{k_{1} x_{n}} \sin \theta\left(x_{n}\right)-2 k_{1} e^{k_{1} x_{n}} \cos \theta\left(x_{n}\right)-2 k_{1} R\left(x_{n}\right) \sin \theta\left(x_{n}\right)\right.
\end{array}\right]
$$


Putting (19) and (23) in (24) we have:
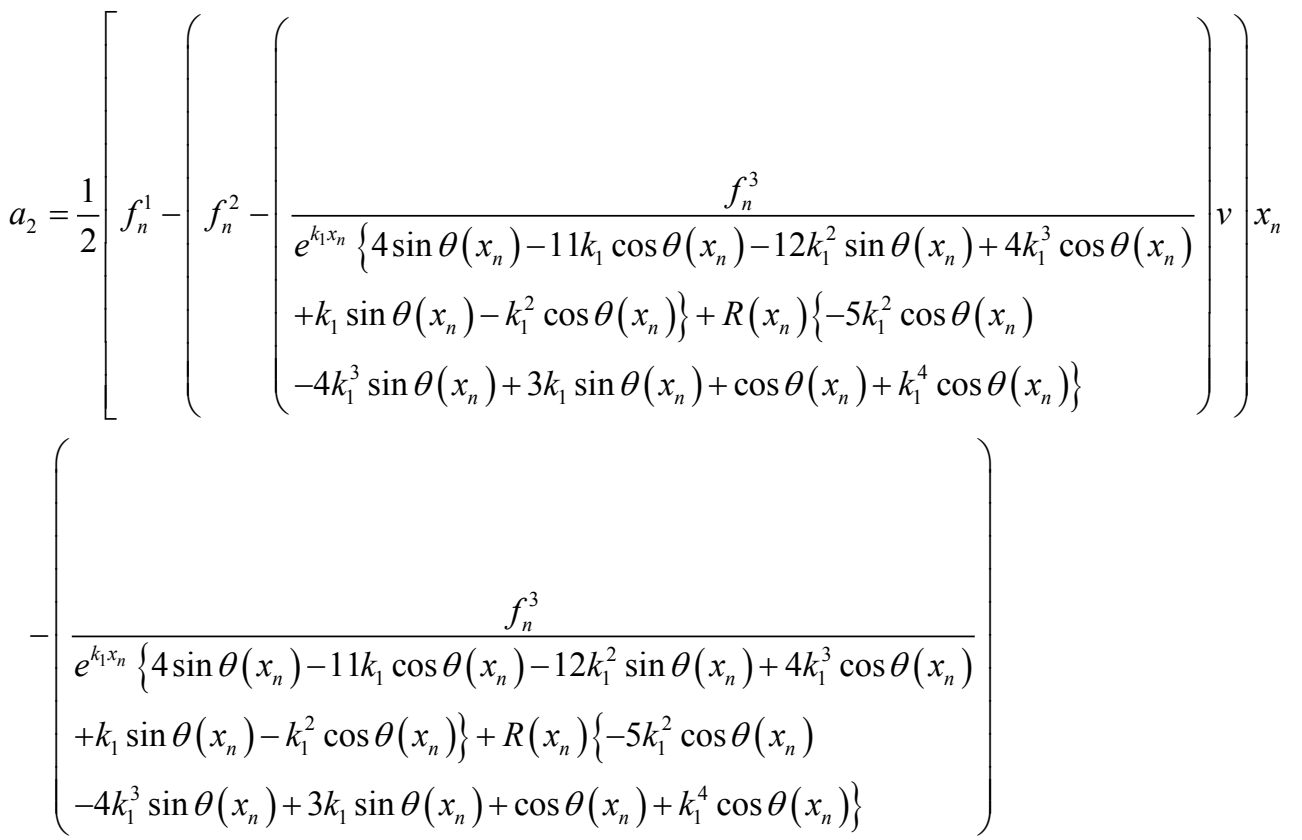

$$
\times\left\{-2 e^{k_{1} x_{n}} \sin \theta\left(x_{n}\right)-2 k_{1} e^{k_{1} x_{n}} \cos \theta\left(x_{n}\right)-2 k_{1} R\left(x_{n}\right) \sin \theta\left(x_{n}\right)\right.
$$

$\left.+k_{1}^{2} R\left(x_{n}\right) \cos \theta\left(x_{n}\right)-R\left(x_{n}\right) \cos \theta\left(x_{n}\right)\right\}$

From (13) we have:

$$
a_{1}=f_{n}-2 a_{2} x_{n}-3 a_{3} x_{n}^{2}-b\left[e^{k_{1} x_{n}} \cos \theta\left(x_{n}\right)+k_{1} R\left(x_{n}\right) \cos \theta\left(x_{n}\right)-R\left(x_{n}\right) \sin \theta\left(x_{n}\right)\right]
$$

Putting (19), (23) and (25) in (26), we have:

$$
\begin{aligned}
& a_{1}=f_{n}-\left(\left[\begin{array}{l}
-\left[f_{n}^{2}-\left\{-3 e^{k_{1} x_{n}} \cos \theta\left(x_{n}\right)-6 k_{1} e^{k_{1} x_{n}} \sin \theta\left(x_{n}\right)+3 k_{1}^{2} e^{k_{1} x_{n}} \cos \theta\left(x_{n}\right)\right.\right. \\
\left.\left.-3 k_{1}^{2} R\left(x_{n}\right) \sin \theta\left(x_{n}\right)-2 k_{1} R\left(x_{n}\right) \cos \theta\left(x_{n}\right)\right\} f_{n}^{3}\right] \\
e^{k_{1} x_{n}}\left\{4 \sin \theta\left(x_{n}\right)-11 k_{1} \cos \theta\left(x_{n}\right)-12 k_{1}^{2} \sin \theta\left(x_{n}\right)+4 k_{1}^{3} \cos \theta\left(x_{n}\right)\right. \\
\left.+k_{1} \sin \theta\left(x_{n}\right)-k_{1}^{2} \cos \theta\left(x_{n}\right)\right\}+R\left(x_{n}\right)\left\{-5 k_{1}^{2} \cos \theta\left(x_{n}\right)\right. \\
\left.-4 k_{1}^{3} \sin \theta\left(x_{n}\right)+3 k_{1} \sin \theta\left(x_{n}\right)+\cos \theta\left(x_{n}\right)+k_{1}^{4} \cos \theta\left(x_{n}\right)\right\}
\end{array}\right] x_{n}\right. \\
& \left\{-2 e^{k_{1} x_{n}} \sin \theta\left(x_{n}\right)-2 k_{1} e^{k_{1} x_{n}} \cos \theta\left(x_{n}\right)-2 k_{1} R\left(x_{n}\right) \sin \theta\left(x_{n}\right)\right. \\
& -\frac{\left.+k_{1}^{2} R\left(x_{n}\right) \cos \theta\left(x_{n}\right)-R\left(x_{n}\right) \cos \theta\left(x_{n}\right)\right\}}{e^{k_{1} x_{n}}\left\{4 \sin \theta\left(x_{n}\right)-11 k_{1} \cos \theta\left(x_{n}\right)-12 k_{1}^{2} \sin \theta\left(x_{n}\right)+4 k_{1}^{3} \cos \theta\left(x_{n}\right)\right.} x_{n} \\
& \left.+k_{1} \sin \theta\left(x_{n}\right)-k_{1}^{2} \cos \theta\left(x_{n}\right)\right\}+R\left(x_{n}\right)\left\{-5 k_{1}^{2} \cos \theta\left(x_{n}\right)\right. \\
& \left.-4 k_{1}^{3} \sin \theta\left(x_{n}\right)+3 k_{1} \sin \theta\left(x_{n}\right)+\cos \theta\left(x_{n}\right)+k_{1}^{4} \cos \theta\left(x_{n}\right)\right\}
\end{aligned}
$$




$$
\begin{aligned}
& -\frac{1}{2}\left[\begin{array}{c}
\left\{-3 e^{k_{1} x_{n}} \cos \theta\left(x_{n}\right)-6 k_{1} e^{k_{1} x_{n}} \sin \theta\left(x_{n}\right)+3 k_{1}^{2} e^{k_{1} x_{n}} \cos \theta\left(x_{n}\right)\right. \\
f_{n}^{2}-\frac{\left.-3 k_{1}^{2} R\left(x_{n}\right) \sin \theta\left(x_{n}\right)-2 k_{1} R\left(x_{n}\right) \cos \theta\left(x_{n}\right)\right\}}{e^{k_{1} x_{n}}\left\{4 \sin \theta\left(x_{n}\right)-11 k_{1} \cos \theta\left(x_{n}\right)-12 k_{1}^{2} \sin \theta\left(x_{n}\right)+4 k_{1}^{3} \cos \theta\left(x_{n}\right)\right.} \\
\left.+k_{1} \sin \theta\left(x_{n}\right)-k_{1}^{2} \cos \theta\left(x_{n}\right)\right\}+R\left(x_{n}\right)\left\{-5 k_{1}^{2} \cos \theta\left(x_{n}\right)\right. \\
\left.-4 k_{1}^{3} \sin \theta\left(x_{n}\right)+3 k_{1} \sin \theta\left(x_{n}\right)+\cos \theta\left(x_{n}\right)+k_{1}^{4} \cos \theta\left(x_{n}\right)\right\}
\end{array}\right] x_{n}^{2} \\
& -\left(\begin{array}{l}
\frac{f_{n}^{3}}{e^{k_{1} x_{n}}\left\{4 \sin \theta\left(x_{n}\right)-11 k_{1} \cos \theta\left(x_{n}\right)-12 k_{1}^{2} \sin \theta\left(x_{n}\right)+4 k_{1}^{3} \cos \theta\left(x_{n}\right)\right.} \\
\left.+k_{1} \sin \theta\left(x_{n}\right)-k_{1}^{2} \cos \theta\left(x_{n}\right)\right\}+R\left(x_{n}\right)\left\{-5 k_{1}^{2} \cos \theta\left(x_{n}\right)\right. \\
\left.-4 k_{1}^{3} \sin \theta\left(x_{n}\right)+3 k_{1} \sin \theta\left(x_{n}\right)+\cos \theta\left(x_{n}\right)+k_{1}^{4} \cos \theta\left(x_{n}\right)\right\}
\end{array}\right) \\
& \times\left[e^{k_{1} x_{n}} \cos \theta\left(x_{n}\right)+k_{1} R\left(x_{n}\right) \cos \theta\left(x_{n}\right)-R\left(x_{n}\right) \sin \theta\left(x_{n}\right)\right]
\end{aligned}
$$

For preservative of the scheme, then we can write the new scheme in a more compact form as:

$$
\begin{aligned}
y_{n+1}= & y_{n}+a_{1} h+a^{2} a_{2}(2 n+1)+h^{3} a_{3}\left(3 n^{2}+3 n+1\right) \\
& +b R_{n}\left[h e^{k_{1}} h\left(\cos \theta_{n} \cos k_{2} h-\sin \theta_{n} \sin k_{2} h\right)-\cos \theta_{n}\right]
\end{aligned}
$$

Putting $a_{1}, a_{2}$ and $a_{3}$ as derived above, we arrived at a new scheme. But to test the scheme, we shall proceed to write a programme which will command the scheme to solve some firs order differential equations.

\section{Implementation of the Scheem}

PROBLEM 1. A spring with a mass of $2 \mathrm{~kg}$ has natural length $\mathrm{m}$. A force of $5 \mathrm{~N}$ is required to maintain it stretched to a length of $\mathrm{m}$. If the spring is stretched to a length of $\mathrm{m}$ and then released with initial velocity 0 , find the position of the mass at any time.

$$
k(0.2)=25.6
$$

$$
\text { So, } k=\frac{25.6}{0.2}=128 \text {. }
$$

Using this value of the spring constant $k$, together with $m=2$ then, we have

$$
\begin{gathered}
2 \frac{\mathrm{d}^{2} x}{\mathrm{~d} t^{2}}+128 x=0 \\
x(t)=c_{1} \cos 8 t+c_{2} \sin 8 t \\
x^{\prime}(t)=-8 c_{1} \sin 8 t+8 c_{2} \cos 8 t
\end{gathered}
$$

Since the initial velocity is given as $x^{\prime}(0)=0$, we have $c_{2}=0$ and so the solution is 


$$
x(t)=\frac{1}{5} \cos 8 t
$$

The result compared favourably with the theoretical solutions.

PROBLEM 2:

Suppose that the spring of Problem 1 is immersed in a fluid with damping constant. Find the position of the mass at any time if it starts from the equilibrium Position and is given a push to start it with an initial velocity of $\mathrm{m} / \mathrm{s}$.

Mathematical Interpretation of the Problem

From Problem 1, the mass is $m=2$ and the spring constant is $k=128$, so the differential Equation (3) becomes

$$
2 \frac{\mathrm{d}^{2} x}{\mathrm{~d} t^{2}}+40 \frac{\mathrm{d} x}{\mathrm{~d} t}+128 x=0
$$

or

$$
\begin{aligned}
& \frac{\mathrm{d}^{2} x}{\mathrm{~d} t^{2}}+20 \frac{\mathrm{d} x}{\mathrm{~d} t}+64 x=0 \\
& x(t)=c_{1} e^{-4 t}+c_{2} e^{-16 t}
\end{aligned}
$$

We are given that $x(0)=0, c_{1}+c_{2}=0$. Differentiating, we get

$$
x^{\prime}(t)=-4 c_{1} e^{-4 t}+16 c_{2} e^{-16 t}
$$

So

$$
x^{\prime}(0)=-4 c_{1}+16 c_{2}=0.6
$$

Since $c_{2}=-c_{1}$, this gives $12 c_{1}=0.6$ or $c_{1}=0.05$. Therefore

$$
x=0.05\left(e^{-4 t}-e^{-16 t}\right)
$$

The result compares favourably with the theoretical solutions.

The result compares favourably with the theoretical solutions.

\section{Conclusions}

The procedure for development and implementation of a numerical algorithm has been examined in this work. The method employed a basis function for approximation. The scheme that formed the derived method was implemented to test the accuracy. The method gave a numerical algorithm capable of solving second order problems of ordinary differential equations. The new numerical algorithm scheme was employed to solve second order initial value problems of ordinary differential equations problems. The computations were carried out in computer codes. Tables 1-4 represent the numerical results of the examples at various values of step size $(\mathrm{H})$. From the tables, it could be concluded that a smaller value of $H$, gives a better result. Figures $1-4$ readily show the comparison of the newly derived method and the theoretical solution. Since the results obtained compare favorably with the theoretical, this shows the accuracy of the 
new method and so can be said to be approximately correct.

Table 1. Numerical results of problem 1 at $H=0.01$.

\begin{tabular}{|c|c|c|c|}
\hline$X(n)$ & THEORITICAL SOLUTION & OLUBUNMI & TRUN \\
\hline 0.00000000 & 0.20000000 & 0.20000000 & 0.00000000 \\
\hline 0.01000000 & 0.19936034 & 0.20000000 & 0.00063966 \\
\hline 0.02000000 & 0.19744547 & 0.19999953 & 0.00255406 \\
\hline 0.03000000 & 0.19426760 & 0.19626706 & 0.00199946 \\
\hline 0.04000000 & 0.18984708 & 0.19087657 & 0.00102949 \\
\hline 0.05000000 & 0.18421221 & 0.18522314 & 0.00101093 \\
\hline 0.06000000 & 0.17739899 & 0,17885647 & 0.00145748 \\
\hline 0.07000000 & 0.16945103 & 0.17057349 & 0.00112246 \\
\hline 0.08000000 & 0.16041915 & 0.16179043 & 0.00137128 \\
\hline 0.09000000 & 0.15036115 & 0.15114648 & 0.00078533 \\
\hline 0.09999999 & 0.13934135 & 0.14011432 & 0.00077297 \\
\hline 0.11000000 & 0.12743023 & 0.12878653 & 0.0013563 \\
\hline 0.12000000 & 0.11470401 & 0.11534238 & 0.00063837 \\
\hline 0.13000000 & 0.10124406 & 0.10815221 & 0.00690815 \\
\hline 0.14000000 & 0.08713649 & 0.08937685 & 0.00224036 \\
\hline 0.14999999 & 0.07247157 & 0.07387969 & 0.00140812 \\
\hline 0.16000000 & 0.05734305 & 0.05876521 & 0.00142216 \\
\hline 0.17000000 & 0.04184773 & 0.04497863 & 0.0031309 \\
\hline 0.17999999 & 0.02608475 & 0.02765649 & 0.00157174 \\
\hline 0.19000000 & 0.01015490 & 0.01217869 & 0.00202379 \\
\hline 0.19999999 & 0.00583989 & 0.00592321 & $8.332 \mathrm{E}-05$ \\
\hline
\end{tabular}

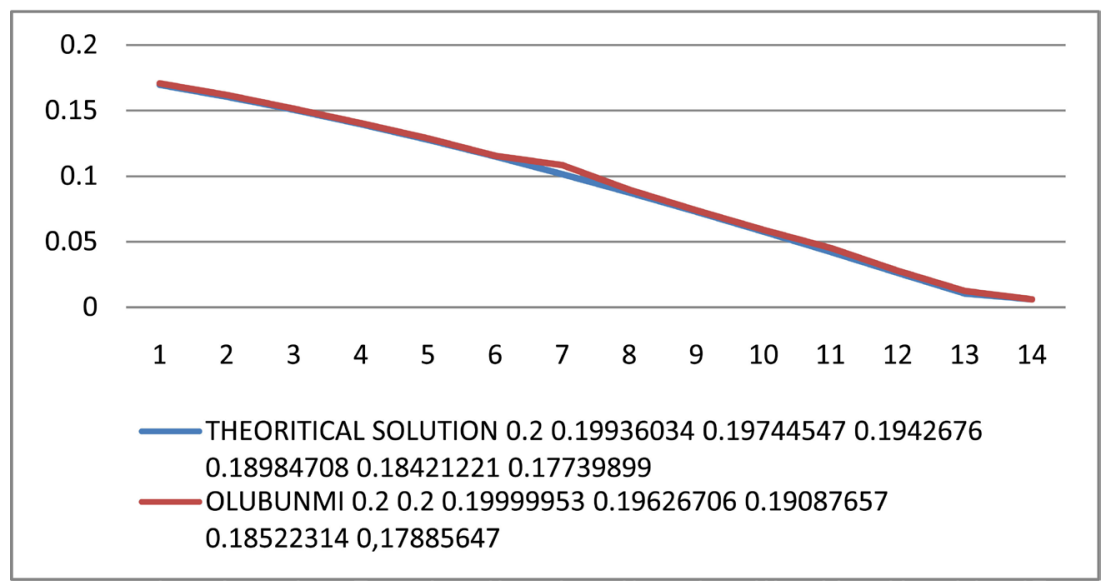

Figure 1. Graphical presentation of problem 1. 
Table 2. Numerical results of problem 1 at $H=0.001$.

\begin{tabular}{|c|c|c|c|}
\hline$X(n)$ & THEORETICAL SOLUTION & OLUBUNMI & TRUN \\
\hline 0.00000000 & 0.20000000 & 0.20000000 & 0.00000000 \\
\hline 0.00100000 & 0.19999361 & 0.20000000 & 0.00000639 \\
\hline 0.00200000 & 0.19997440 & 0.19998160 & 0.00000720 \\
\hline 0.00300000 & 0.19994241 & 0.19995000 & 0.00000759 \\
\hline 0.00400000 & 0.19989762 & 0.19990601 & 0.00000839 \\
\hline 0.00500000 & 0.19984002 & 0.19984885 & 0.00000883 \\
\hline 0.00600000 & 0.19976965 & 0.19977901 & 0.00000936 \\
\hline 0.00700000 & 0.19968648 & 0.19969999 & 0.00001351 \\
\hline 0.00800000 & 0.19959055 & 0.19960510 & 0.00001455 \\
\hline 0.00900000 & 0.19948183 & 0.19949996 & 0.00001813 \\
\hline 0.01000000 & 0.19936034 & 0.19937993 & 0.00001959 \\
\hline 0.01100000 & 0.19922610 & 0.19924990 & 0.00002380 \\
\hline 0.01200000 & 0.19907911 & 0.19910485 & 0.00002574 \\
\hline 0.01300000 & 0.19891937 & 0.19894679 & 0.00002742 \\
\hline 0.01400000 & 0.19874692 & 0.19877972 & 0.00003280 \\
\hline 0.01500000 & 0.19856173 & 0.19859963 & 0.00003790 \\
\hline 0.01600000 & 0.19836384 & 0.19840453 & 0.00004069 \\
\hline 0.01700000 & 0.19815326 & 0.19819939 & 0.00004613 \\
\hline 0.01800000 & 0.19792998 & 0.19797923 & 0.00004925 \\
\hline 0.01900000 & 0.19769405 & 0.19774904 & 0.00005499 \\
\hline 0.02000000 & 0.19744545 & 0.19750381 & 0.00005836 \\
\hline
\end{tabular}

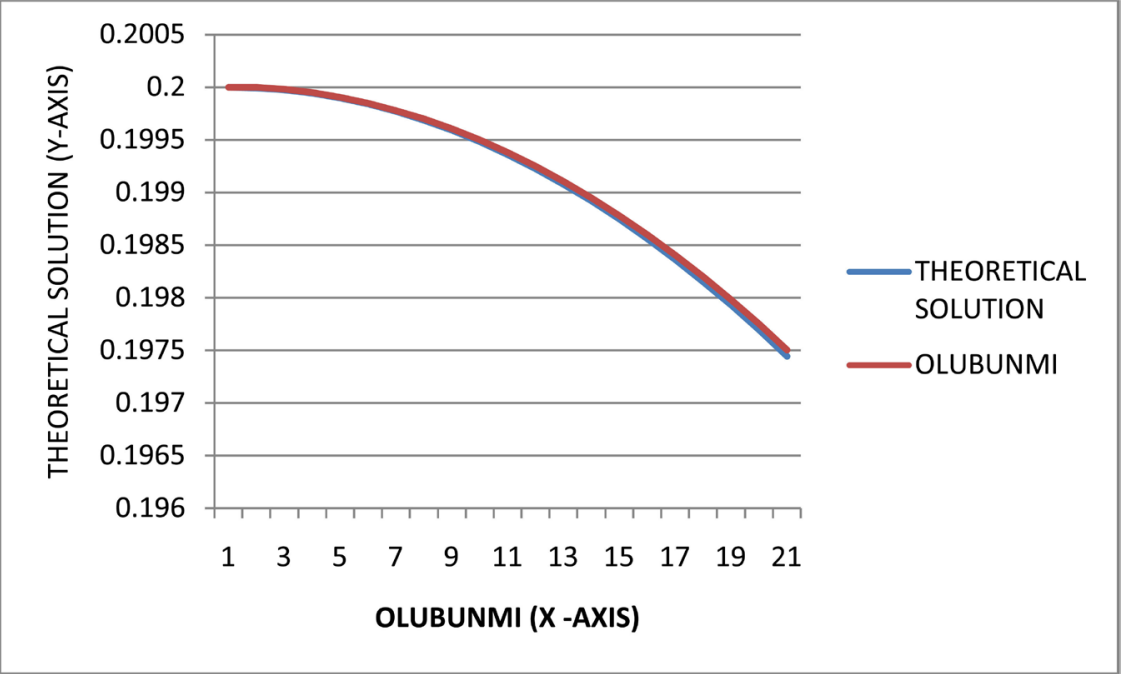

Figure 2. Graphical presentation of problem 1. 
Table 3. Numerical results of problem 2 at $H=0.001$.

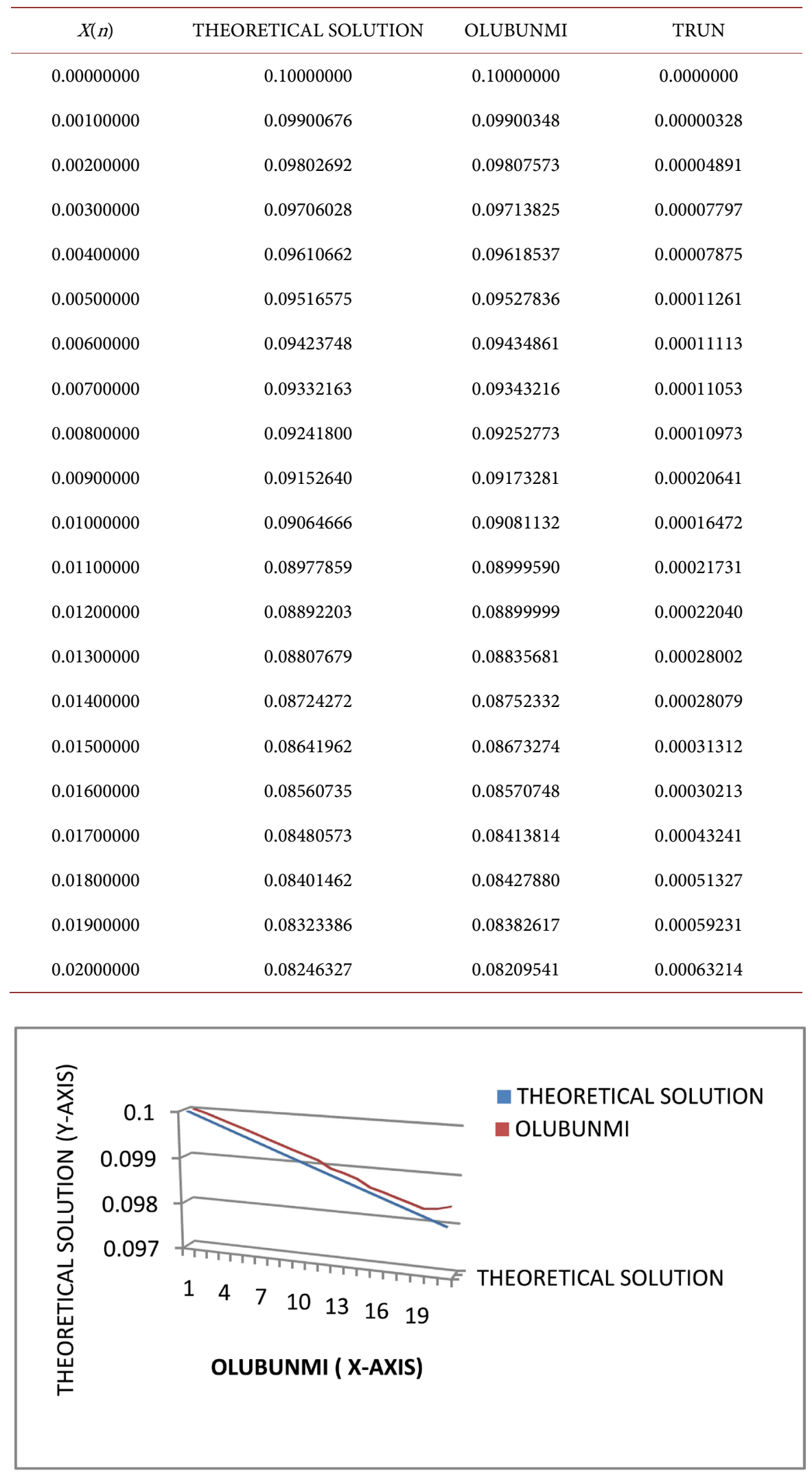

Figure 3. Graphical presentation of problem 2. 
Table 4. Numerical results of problem 2 at $H=0.0001$.

\begin{tabular}{|c|c|c|c|}
\hline$X(n)$ & THEORETICAL SOLUTION & OLUBUNMI & TRUN \\
\hline 0.00000000 & 0.10000000 & 0.10000000 & 0.00000000 \\
\hline 0.00010000 & 0.09990007 & 0.09992720 & 0.00002713 \\
\hline 0.00020000 & 0.09980027 & 0.09983454 & 0.00003427 \\
\hline 0.00030000 & 0.09970061 & 0.09974292 & 0.00004231 \\
\hline 0.00040000 & 0.09960109 & 0.09965531 & 0.00005422 \\
\hline 0.00050000 & 0.09950170 & 0.09956093 & 0.00005923 \\
\hline 0.00060000 & 0.09940244 & 0.09946347 & 0.00006123 \\
\hline 0.00070000 & 0.09930332 & 0.09937088 & 0.00006676 \\
\hline 0.00080000 & 0.09920434 & 0.09927559 & 0.00007125 \\
\hline 0.00090000 & 0.09910548 & 0.09918759 & 0.00008211 \\
\hline 0.00100000 & 0.09900676 & 0.09909777 & 0.00009101 \\
\hline 0.00110000 & 0.09890819 & 0.09894247 & 0.00010113 \\
\hline 0.00120000 & 0.09880973 & 0.09886919 & 0.00013274 \\
\hline 0.00130000 & 0.09871142 & 0.09877561 & 0.00015777 \\
\hline 0.00140000 & 0.09861323 & 0.09861727 & 0.00016238 \\
\hline 0.00150000 & 0.09851518 & 0.09854087 & 0.00018621 \\
\hline 0.00160000 & 0.09841727 & 0.09845110 & 0.00020000 \\
\hline 0.00170000 & 0.09831949 & 0.09836652 & 0.00022135 \\
\hline 0.00180000 & 0.09822183 & 0.09827469 & 0.00022927 \\
\hline 0.00190000 & 0.09812431 & 0.09830139 & 0.00024221 \\
\hline 0.00200000 & 0.09802692 & 0.09837561 & 0.00027725 \\
\hline
\end{tabular}

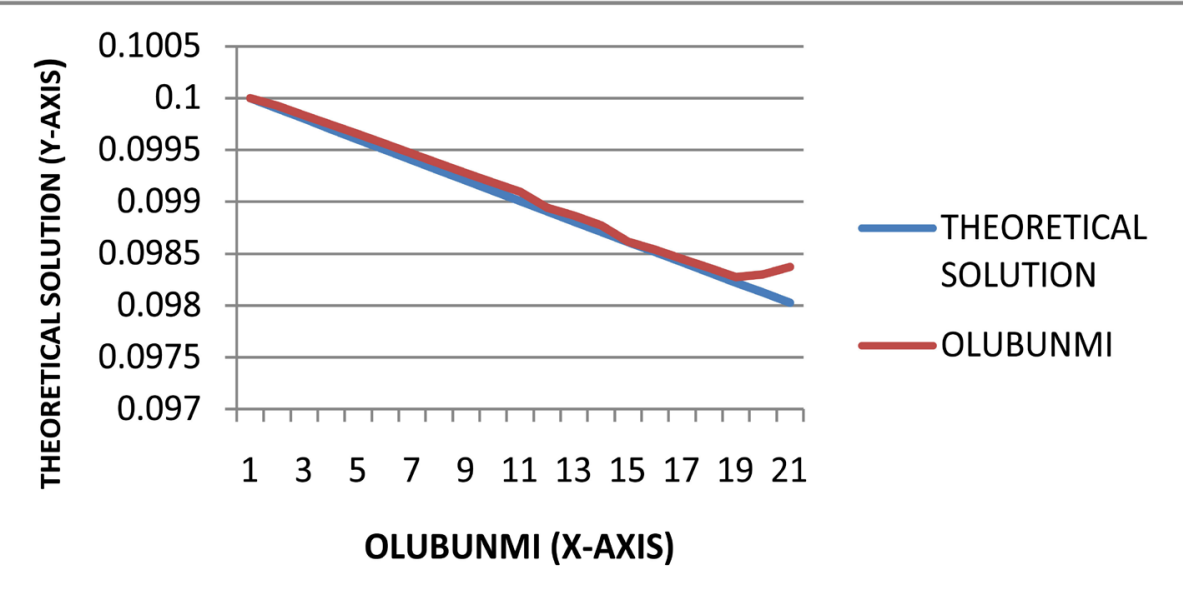

Figure 4. Graphical presentation of problem 2. 
In our subsequent research, we shall pay more attention on the implementation of this new scheme to solve some higher order initial value problems of ordinary differential equation and also compare the results with the existing methods and thereafter we examine the characteristics properties such as the stability, convergence, accuracy and consistency of the scheme.

\section{Conflicts of Interest}

The authors declare no conflicts of interest regarding the publication of this paper.

\section{References}

[1] Fatunla, S.O. (1987) An Implicit Two-Point Numerical Integration Formula for Linear and Non-Linear Stiff System of ODEs. Mathematics of Computation, 32, 1-11. https://doi.org/10.1090/S0025-5718-1978-0474830-0

[2] Ibijola, E.A. (1997) A New Numerical Scheme for the Solution of Initial Value Problem (IVPs). Ph.D. Thesis, University of Benin, Nigeria.

[3] Ibijola, E.A. (1998) On the Convergence, Consistency and Stability of a One-Step Method for Integration of ODEs. International Journal of Computer Mathematics, 73, 261-277. https://doi.org/10.1080/00207169908804894

[4] Obayomi, A.A. (2012) A Set of Non-Standard Finite Difference Schemes for the Solution of an Equation of the Type $y^{\prime}=y\left(1-y^{n}\right)$. International Journal of Pure and Applied Sciences and Technology, 12, 34-42.

[5] Ogunrinde, R.B. (2010) A New Numerical Scheme for the Solution of Initial Value Problems in Ordinary Differential Equations. Ph. D. Thesis, University of Ado Ekiti, Nigeria.

[6] Ogunrinde, R.B. (2013) On Some Models Based on First Order Differential Equations. American Journal of Scientific and Industrial Research, 4, 288-293. https://doi.org/10.5251/ajsir.2013.4.3.288.293

[7] Ogunrinde, R.B. and Lebile, O. (2015) On Mathematical Model of Traffic Control. Mathematical Theory and Modeling, 5, 58-68.

[8] Ogunrinde, R.B. (2016) On Error Analysis Comparison of Some Numerical Experimental Results.

[9] Ogunrinde, R.B., et al. (2012) On Some Numerical Methods for Solving Initial Value Problems in Ordinary Differential Equation. International Organization of Scientific Research (IOSR-JM), 1, 25-31. 